\title{
Interactions of like-charged rods at low temperatures: Analytical theory vs. simulations
}

\author{
A. Arnold ${ }^{1,3, a}$ and C. Holm $2,3, \mathrm{~b}$ \\ 1 FOM-Institute AMOLF, Kruislaan 407, 1098 SJ Amsterdam, The Netherlands \\ 2 Frankfurt Institute for Advanced Studies, JW Goethe Universität, Routh-Moufang-Straße 1, 60438 Frankfurt, Germany \\ 3 Max-Planck-Institut für Polymerforschung, Ackermannweg 10, 55128 Mainz, Germany
}

Received 21 December 2007

Published online: 21 July 2008 - (C) EDP Sciences / Società Italiana di Fisica / Springer-Verlag 2008

\begin{abstract}
We investigate a system consisting of two like-charged infinitely long rods and neutralizing counterions at low temperatures, using both analytic theory and simulations. With some reasonable approximations we can analytically solve for several ground-state structures of the model, starting with states where all counterions are lined up in the gap between the rods, over planar configurations, where the counterions are divided up into a fraction which resides between the rods, and counterions which are located on the outer surfaces, up to configurations which cover the full rod surfaces. Using parallel tempering simulations, we are able to study the system over a wide range of temperatures. At low temperatures we find good agreement with our $T=0$ results. At higher temperatures, the strong coupling (SC) theory delivers qualitatively better results. We furthermore demonstrate that for the SC theory and our ground-state approximations to yield quantitative agreement, three parameters are required to be large, the strong-coupling parameter $\Xi$, the Rouzina-Bloomfield parameter, and the ratio of the average distance of the counterions to the radius of the rods. In the case of the latter ratio being small, our $T=0$ results show better agreement with the simulation data at very low temperatures.
\end{abstract}

PACS. 87.15.-v Biomolecules: structure and physical properties - 87.15.N- Properties of solutions of macromolecules

\section{Introduction}

In a polar solvent, macromolecules possessing dissociable groups can acquire a charge by ionic dissociation. In this way, charged polymers, called polyelectrolytes, or charged macromolecules in general, are surrounded by a so-called "double layer" of oppositely charged counterions. It is well known that sufficiently strong electrostatic interactions lead to behavior which cannot be described on the mean-field level. The most prominent example is like-charged attraction: it can be proven rigorously that the standard mean-field Poisson-Boltzmann theory will always predict repulsion between like-charged macroions, regardless of their shape [1-4]. Nevertheless attractive interactions for rod-like macroions have been confirmed by a large number of computer simulations [5-16], and experiments have shown that DNA, a stiff, highly negatively charged polyelectrolyte, can be condensed by multivalent counterions [17-19]. This correlation-induced attraction is for instance believed to be important for the compaction of DNA inside viral capsids [20,21]. In this article we deal

a e-mail: arnold@amolf.nl

b e-mail: c.holm@fias.uni-frankfurt.de with the case of two infinitely long, charged rigid rods, which can be regarded as simple DNA models or more generally as a good approximation for studying the ion distribution around semi-flexible polyelectrolytes.

The experimental as well as the theoretical observations indicate that attraction of like-charged objects occurs in systems which interact via strong Coulomb interactions, i.e. when multivalent counterions are present, the macroions are highly charged, or at high Bjerrum lengths. The Bjerrum length $l_{\mathrm{B}}$ is defined as $l_{\mathrm{B}}=\frac{e^{2}}{4 \pi \epsilon k_{\mathrm{B}} T}$, where $e, \epsilon, k_{\mathrm{B}}, T$ denote the unit charge, dielectric permittivity, Boltzmann constant, and temperature, respectively; therefore, a high Bjerrum length corresponds to a low temperature, or in general a strong Coulomb interaction.

In a previous simulation study [22], we found that for small Bjerrum lengths, the force between the two rods is dominated by their plain repulsion. But already at moderate Bjerrum lengths, attraction sets in, which often goes along with a strong correlation hole in the radial distribution function of the counterions and interlocking patterns along the parallel rods. This suggests that the counterions are starting to freeze into a quasi-crystalline flat pattern in the plane spanned by the two rods. 
The interesting findings of our previous study motivated us to investigate the ground-state properties of the system, which is the topic of the present article. We will show that the ground-state configurations essentially only depend on the geometric parameter $\gamma_{z}$, which is defined as the ratio between the lateral average counterion distance parallel to the rods, and half of the rod distance. For high $\gamma_{z}$, all counterions are lined up equally spaced between the rods, but below a certain value of $\gamma_{z}$, we predict a regime of two-dimensional configurations with interlocking patterns along the inner and outer rod sides. Only for small values of $\gamma_{z}$, the stable configurations are actually three-dimensional, i.e. counterions are found outside the plane spanned by the two rods.

To verify our findings, we performed computer studies using parallel tempering, which vastly extend our previous findings [22]. We compare the results of these simulations both to our $T=0$ approximations as well as to the strong coupling (SC) theory of Moreira and Netz [23,24]. This theory is suited for systems with strong electrostatic coupling, and has been adapted to the case of two charged rods recently $[25,26]$. The theory has been shown to be a good approximation only for large Rouzina-Bloomfield parameter $\gamma_{\mathrm{RB}}$, which is defined similar to $\gamma_{z}$ as the ratio between the lateral average counterion distance parallel to the rods, and the surface-to-surface distance of the rods.

The force between the two rods is indeed described well by the SC theory for a wide range of temperatures, but only above a critical value for $\gamma_{z}$. For values of $\gamma_{z}$, for which the $T=0$ theory predicts ions to be located also outside the gap, we find strong deviations between simulations and the $\mathrm{SC}$ prediction at all temperatures. We therefore conclude that the SC theory is quantitatively useful only if, in addition to a large value of $\gamma_{\mathrm{RB}}$, also the parameter $\gamma_{z}$ has to be sufficiently large.

\section{Approximate ground states}

We start with an analytical investigation of the ground states of a system consisting of two infinitely long parallel rods, which are neutralized by point-like charged particles or counterions. As we show below, these rods attract, and therefore the equilibrium orientation of the two rods is parallel, which we assume to hold in the following. Furthermore we assume that the rods both have a line charge density of $\tau<0$, a radius $R$ and a surface-to-surface distance of $\Delta$, and that the counterions have a charge of $q>0$ (see Fig. 1). The total energy of the system is given by

$$
\begin{aligned}
\tilde{E}= & \sum_{i<j} \frac{q^{2}}{\left|r_{i}-r_{j}\right|}+q \tau \sum_{i}\left\{\log \left[\left(x_{i}-R-\Delta / 2\right)^{2}+y_{i}^{2}\right]\right. \\
& \left.+\log \left[\left(x_{i}+R+\Delta / 2\right)^{2}+y_{i}^{2}\right]-4 \log R\right\} \\
= & q \tau \sum_{i<j} \frac{\gamma_{z}}{\left|\tilde{r}_{i}-\tilde{r}_{j}\right|}+q \tau \sum_{i}\left\{\log \left[\left(\tilde{x}_{i}-1-\tilde{\Delta} / 2\right)^{2}+\tilde{y}_{i}^{2}\right]\right. \\
& \left.+\log \left[\left(\tilde{x}_{i}+1+\tilde{\Delta} / 2\right)^{2}+\tilde{y}_{i}^{2}\right]\right\}
\end{aligned}
$$

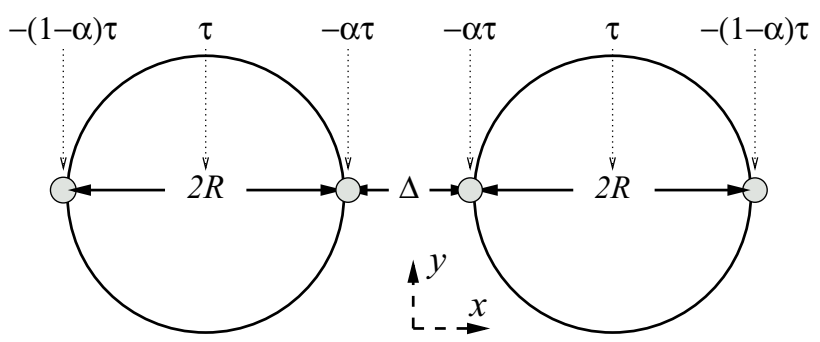

Fig. 1. Schematic view of the two-rod system, with the view axis parallel to the rod axis. The large circles represent the two rods of radius $R$ and line charge density $\tau$ at a surface-tosurface distance $\Delta$. For the analytic calculations, it is assumed that the particles form rods at the four positions marked by small gray circles, with line charge densities given at the top of the graph. The parameter $\alpha$ measures the fraction of counterions located in the gap. The dashed arrows indicate the $x$ - and $y$-axis as used in the text, the $z$-axis is assumed to be parallel to the rods.

where the tilde denotes a rescaling of the coordinates by the rod radius $R$ and the energy by $e^{2} /(4 \pi \epsilon)$.

$$
\gamma_{z}=q /(\tau R)
$$

measures the ratio between the average charge separation parallel to the rods and the macroion-rod radius. $\gamma_{z}$ and $\tilde{\Delta}$ are the only parameters describing the geometry of the system, and $q \tau e^{2} /(4 \pi \epsilon)\left(=l_{\mathrm{B}} q \tau k_{\mathrm{B}} T\right.$ at finite temperature) determines the energy scale.

Finding the ground states of this system is nevertheless a formidable task, therefore we have to apply some approximations that will be proven to be reasonable later by comparing our findings to results obtained via computer simulation. The situation is somewhat improved by Earnshaw's theorem which states that a collection of point charges cannot be kept in a static configuration by purely electrostatic interactions [27]. Since the rods are the only objects with finite extension, this means that they either have to repel each other and escape to infinity, or they have to be located surface to surface $(\tilde{\Delta}=0)$ in the ground state, with the counterions being distributed on the rod surfaces. Our calculations and simulations however show that at least at short distances the rods attract, while at large distances, they barely interact. Therefore, the ground state of the system is the one with the two rods in contact. Moreover the attraction will rotate the rods into a parallel aligned position, and we can restrict our investigation to two parallel rods in contact. However, we will not assume $\tilde{\Delta}=0$, but only that $\tilde{\Delta}$ is small. This allows to compare to numerical results for low, non-zero temperatures, at which the rods are not in direct contact in general.

For a single counterion, there are only two symmetric electrostatic energy minima, located in the small gap between the rods on one of the rod surfaces. So each of the counterions individually wants to be located in the gap. However, with decreasing $\gamma_{z}$, resulting in an increase in counterions, the gap becomes more and more populated, and sooner or later the repulsion due to the many-body 


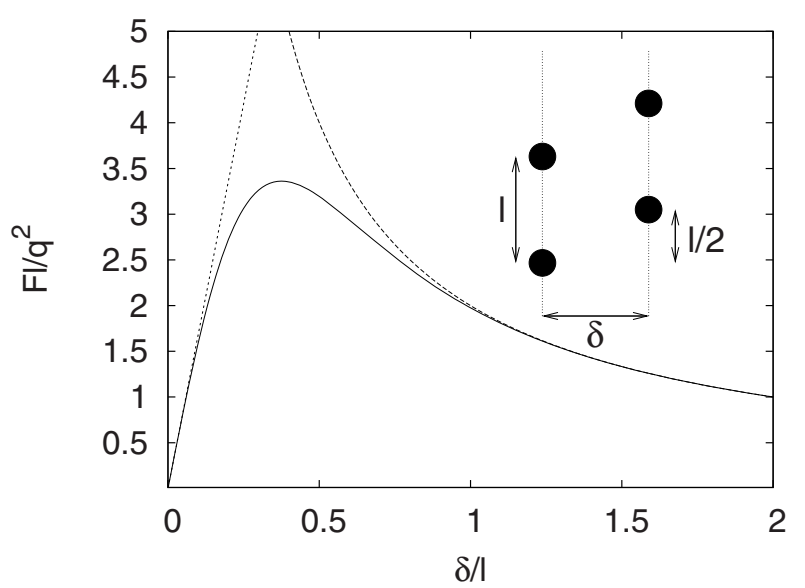

Fig. 2. Force between two interleaved regular charge grids as depicted in the inset. The solid line shows the numeric result obtained from MMM1D, the dotted lines the approximations given by equations (7) (right branch for large $\delta$ ) and (8) (left branch for small $\delta$ ).

interactions of the counterions pushes some counterions out of the gap. To minimize the repulsion exerted by the counterions remaining in the gap, the expelled counterions will jump to the outer sides of the rods. Further decreasing $\gamma_{z}$ will force more and more particles out of the plane spanned by by the rods, until the particles form a nearly homogeneous layer around the rods, which can be described by Wigner-Seitz cells.

Therefore we restrict ourselves in the analytical treatment to only those configurations with particles in the gap and on the opposite sides of the rods, and determining the onset of the transition from planar to non-planar configurations. This leaves four positions for the counterions in the $x y$-plane perpendicular to the rods, namely either on the outer sides of the rods or on one of the rod surfaces in the gap (see Fig. 1). The total system therefore essentially consists of six rods, of which two are homogeneous line charges representing the macroions, and four consist of discrete point charges representing the particles. To avoid confusion, we will call them macroion-rods and particle-rods, respectively, in the following.

We now define $\alpha$ such that each of the inner particlerods, i.e. the rods formed by the counterions in the gap, has a line charge density of $\alpha \tau$, while the two particle-rods on the opposite sides have line charge density $(1-\alpha) \tau$, due to symmetry and charge neutrality. A value of $\alpha=1$ means that all counterions are located in the gap, while $\alpha=0$ is the case where all counterions are on the outer sides of the rods.

The interactions of the particle-rods are highly complex, since for arbitrary $\alpha$ the particles on the inner and outer particle-rods will have different particle spacings. However, on larger scales, the particles will form regular grids. For a perfect regular grid, as depicted in Figure 2, we can approximate the interaction well by analytical expressions both for small and large distances, as we will show now.

\subsection{Energy approximations}

For the calculation of the approximate energies, we will use the formulas of MMM1D [28], which in its original formulation is a method to calculate the electrostatic energy in three-dimensional systems with one periodic coordinate, and which is used to perform the computer simulations presented later. Here, however, the MMM1D formulas will also be used to analytically calculate the energies of the approximate ground states. To this aim, we have to quickly repeat some of the properties of MMM1D. It is assumed that the system is periodic along only the $z$-axis, with a periodicity length of $\lambda_{z}$. For convenience, we define the inverse periodicity length $u_{z}=1 / \lambda_{z}$. The MMM1D method allows to calculate the total rescaled system energy per length $\lambda_{z}$ of a system of $N$ charges as a sum over an effective pair potential. For this effective potential, MMM1D provides two well-convergent series, one for particles with small lateral distance $\rho_{i j}$ and one for $\rho>0$. Both series reveal some important properties of the electrostatic interaction: at large distances $\rho>\lambda_{z}$, the interaction of two (periodically replicated) charges is essentially rod-like. For $\rho<\frac{1}{2} \lambda_{z}$, we obtain from MMM1D the effective interaction energy of two closely located charges as

$$
\begin{aligned}
-u_{z} \sum_{n \geq 0}\left(\begin{array}{c}
-\frac{1}{2} \\
n
\end{array}\right) & \frac{\psi^{(2 n)}\left(1+u_{z} z\right)+\psi^{(2 n)}\left(1-u_{z} z\right)}{(2 n) !}\left(u_{z} \rho\right)^{2 n} \\
& +\frac{1}{r}-2 u_{z} \gamma
\end{aligned}
$$

where $\psi^{(2 n)}$ denotes the $2 n$-th polygamma function [29], and $\gamma$ Euler's constant. Finally, the effective interaction energy between a charge $q$ and a rod of line charge density $\tau$ is

and

$$
-2 q \tau\left(\log \left[\rho /\left(2 \lambda_{z}\right)\right]+\gamma\right)
$$

$$
-2 \lambda_{z} \tau \tau^{\prime}\left(\log \left[\rho /\left(2 \lambda_{z}\right)\right]+\gamma\right)
$$

for two rods of line charge densities $\tau$ and $\tau^{\prime}$. The forces can be obtained from the energies by simply taking the derivative.

From the effective interaction energies of single particles, we can obtain the interaction energies for a particlerod consisting of charges $q$, regularly distributed with lateral distance $l$. For the interaction with a rod of line charge density $\tau$ at distance $\delta$, we obtain from equation (4)

$$
\tilde{E}_{\mathrm{rp}}(l, \tau, \delta)=-2 \lambda_{z} \frac{q}{l} \tau\left(\log \left[\delta /\left(2 \lambda_{z}\right)\right]+\gamma\right),
$$

which is simply the interaction of two rods of charge densities $\tau$ and $q / l$, independent of the lateral distance between particle-rod and rod.

For the interaction with a second particle-rod with a parallel separation $l^{\prime}$, we obtain

$$
\tilde{E}_{\mathrm{pp}}^{\mathrm{far}}\left(l, l^{\prime}, \delta\right)=\tilde{E}_{\mathrm{rp}}\left(l, q / l^{\prime}, \delta\right),
$$

provided that the lateral distance $\delta$ is larger than $l$ and $l^{\prime}$. In this case, the MMM1D formalism shows that the interaction is essentially rod-like, which is demonstrated in Figure 2. For the interactions of the outer and inner 
particle-rods, we have a lateral distance of $\delta=2 R$ and a charge distance $l \leq q /(2 \tau)$; the approximation therefore holds if $\gamma_{z}=4 l / \delta \leq 8$. On the other hand, we will show that already at $\gamma_{z} \geq 4$ all ions line up between the rods, in which case our calculations are exact. The two outer particle-rods are even further apart.

For the interaction of the two inner particle-rods, we, in contrast, need a formula for $\delta \ll \lambda_{z}$. At close distances, the strong local repulsion will force the two particle-rods to interleave, as depicted in Figure 2. In this case, we can approximate the interaction by the terms of order $\delta^{2}$ or lower of equation (3). We obtain

$$
\tilde{E}_{\mathrm{pp}}^{\mathrm{close}}(l, \delta)=\lambda_{z} \frac{q^{2}}{l^{2}}\left[\xi-2 \log \left(\frac{\lambda_{z}}{l}\right)-\eta\left(\frac{\delta}{l}\right)^{2}\right]
$$

where

$$
\begin{aligned}
& \xi=2-2 \gamma-\psi^{(0)}\left(\frac{1}{2}\right)-\psi^{(0)}\left(\frac{3}{2}\right) \approx 2.77 \\
& \eta=4-\left[\psi^{(2)}\left(\frac{1}{2}\right)+\psi^{(2)}\left(\frac{3}{2}\right)\right] / 4 \approx 8.41 .
\end{aligned}
$$

The validity of this approximation is also demonstrated in Figure 2. It will be used for the interaction of the two inner particle-rods which have a lateral distance $\Delta$. Therefore, the approximation is reasonable for small $\Delta / l=2 \tau \Delta / q=$ $\gamma_{\mathrm{RB}}^{-1}$. In other words, similar to the SC theory, we require a large Rouzina-Bloomfield parameter, that is, larger than $\approx 5$.

The self-energy of a particle-rod can be conveniently derived from the MMM1D self-energy, and is given by

$$
\tilde{E}_{\text {self }}(l)=\frac{\lambda_{z} q^{2}}{l^{2}} \log \frac{\lambda_{z}}{l} .
$$

\subsection{Force between the rods}

We now use the MMM1D formulas to determine under which circumstances certain configurations are stable and which value of $\alpha$ is optimal, i.e. has minimal energy. The stability of the configuration requires that i) the net force between the macroion-rods is attractive, so that they do not move apart, and ii) that a particle in both the outer and the inner particle-rods is in a local energetic minimum, so that none of the particles is able to move. From the stable configurations, we then determine the one with the lowest energy as our approximate ground state. It is not sure that this configuration is a minimum of the original two-rod problem. However, we have argued above why these states should be close to the true ground states, and we will present computer simulation results that confirm our predictions.

First, we calculate the force between two rods in the planar model. For the ground state, we know that the force has to be attractive, i.e. negative; however, for arbitrary $\alpha$, the force may be repulsive as well. There are not only the electrostatic forces acting on the macroion-rods themselves; in addition, the particle-rods exert forces on the macroion-rods in contact, since the outer particle-rods are attracted towards the center, while the inner particle-rods are pushed away from the center. Taking this into account, one finds the total force between the two macroion-rods as

$$
\begin{aligned}
2 \frac{\lambda_{z} \tau^{2}}{R}( & -\frac{2 \alpha}{1+\tilde{\Delta}}+\frac{1+2 \alpha-2 \alpha^{2}}{2+\tilde{\Delta}} \\
& \left.-\frac{2(1-\alpha)}{3+\tilde{\Delta}}+\frac{(1-\alpha)^{2}}{4+\tilde{\Delta}}+\frac{\alpha^{2} \eta \tilde{\Delta}}{\gamma_{z}^{2}}\right)
\end{aligned}
$$

For $\tilde{\Delta}=0$, this reduces to $-2 \lambda_{z} \tau^{2} / R\left(9 \alpha^{2}+10 \alpha-1\right)$, which is negative at least for all values $1 / 10<\alpha \leq 1$, i.e. the rods attract; this also holds for small $\tilde{\Delta}>0$. This shows that, actually, even a relatively small portion of ions between the rods is sufficient to induce attraction.

\subsection{Stability of the particle-rods}

Next we investigate the stability of the particle-rods, i.e., whether the planar configuration is actually stable, or whether counterions would rather like to leave the plane. To this aim, we calculate the second derivative of the electrostatic energy for a single particle moving out of the macroion-rod plane into the $y$-dimension. The particle will move only along the surface of the rod on which it is currently sitting, since the electrostatic energy is minimal there.

In principle, one would also have to look at two or more particles leaving their particle-rods simultaneously, which might be easier; therefore, the true area of stability might be smaller than our prediction. However, the primary driving force for moving a particle out of its particle-rod is the strong interaction with the direct neighbors. This does not decrease much further even if two neighboring counterions move apart, so that the stable area should not change dramatically. In fact, our simulation results will justify this simplification a posteriori.

We assume that a single charge is located initially in one of the particle-rods, and moves by small angle $\delta$ on the surface of its macroion-rod. The interaction of this charge with the other particles in the particle-rod, which it is just leaving, can be readily determined using equation (3) for a single pair of charges with $z=0$ and a periodicity $\lambda_{z}$ equal to the particle spacing $l$ (of course, the $1 / r$ term has to be omitted). The interaction energy between the moving charge and the macroion-rods, as well as its energies with distant particle-rods, can be easily determined from equation (6). In the case that the moving charge is in one of the two inner particle-rods, the interaction with the second particle-rod can be obtained from equation (8).

Combining these contributions, we obtain for an inner particle moving on the surface of a macroion-rod an energy difference of

$$
\begin{aligned}
\Delta \tilde{E}_{\mathrm{i}}= & -q \tau\left(2-\frac{\tilde{\Delta}}{(1+\tilde{\Delta})^{2}}+(1-\alpha)\left(\frac{1+\tilde{\Delta}}{(2+\tilde{\Delta})^{2}}-\frac{3}{4}\right)\right. \\
& \left.+\frac{\alpha^{3}}{\gamma_{z}^{2}}\left[\frac{\psi^{(2)}(1)}{2}-\eta(1+\tilde{\Delta})\right]\right) \delta^{2}+\mathcal{O}\left(\delta^{4}\right),
\end{aligned}
$$


where $\gamma_{z}$ is the geometry parameter introduced in equation (1).

From equation (11), we can obtain an upper limit on $\alpha$. Stability requires that $\delta=0$ is a local minimum, and since $-q \tau>0$, equation (11) gives the following implicit upper limit for $\alpha$ :

$$
\begin{aligned}
\gamma_{z}^{2}[2 & \left.-\frac{\tilde{\Delta}}{(1+\tilde{\Delta})^{2}}+(1-\alpha)\left(\frac{1+\tilde{\Delta}}{(2+\tilde{\Delta})^{2}}-\frac{3}{4}\right)\right] \\
& +\alpha^{3}\left[\frac{\psi^{(2)}(1)}{2}-\eta(1+\tilde{\Delta})\right]>0
\end{aligned}
$$

where $\psi^{(2)}(1) \approx-2.40$, and $\eta \approx 8.41$.

For the stability of the outer particle-rod, we obtain by a similar calculation the following implicit lower limit on $\alpha$ :

$$
\begin{aligned}
& \gamma_{z}^{2}\left[-\frac{2+\tilde{\Delta}}{(3+\tilde{\Delta})^{2}}+\alpha\left[\frac{1}{4}+\frac{1+\tilde{\Delta}}{(2+\tilde{\Delta})^{2}}\right]\right. \\
& \left.\quad+(1-\alpha) \frac{3+\tilde{\Delta}}{(4+\tilde{\Delta})^{2}}\right]+\frac{(1-\alpha)^{3}}{2} \psi^{(2)}(1)>0 .
\end{aligned}
$$

For small $\tilde{\Delta}$, this lower limit on $\alpha$ is stricter than the requirement of attraction between the rods. The combinations of $\alpha$ and $\gamma_{z}$ excluded by equations (12) and (13) are displayed in gray in Figure 3 and following.

\subsection{The energetically optimal $\alpha$}

Since we know now for which values of $\alpha$ the planar configurations are stable, we compare their energies to determine the lowest energy state. To this aim we calculate the energy difference to the $\alpha=1$ state:

$$
\begin{aligned}
\Delta \tilde{E}= & 1 / 2 \tau^{2}\left[-\left(1-\alpha^{2}\right) \xi-\gamma\left(2+4 \alpha-6 \alpha^{2}\right)\right. \\
& +\left(1-\alpha^{2}\right) \log 4+4 \alpha^{2} \log \alpha+2(1-\alpha)^{2} \log (1-\alpha) \\
& -4(1-\alpha) \log (1+\tilde{\Delta})-4(1-\alpha) \alpha \log (2+\tilde{\Delta}) \\
& +4(1-\alpha) \log (3+\tilde{\Delta})-2(1-\alpha)^{2} \log [4+\tilde{\Delta}] \\
& \left.+\left(1-\alpha^{4}\right) \tilde{\Delta}^{2} \eta / \gamma_{z}^{2}+\left(2+4 \alpha-6 \alpha^{2}\right) \log \left(\gamma_{z}\right)\right],
\end{aligned}
$$

where we have inserted the geometry parameter $\gamma_{z}=$ $q /(\tau R)$ and $\tilde{\Delta}=\Delta / R$. The global minimum of $\Delta \tilde{E}$ is either at the boundary $\alpha=1$, with an energy difference of 0 , or where

$$
\begin{aligned}
0= & -\tau^{-2} \partial_{\alpha} \Delta \tilde{E}=1+2 \gamma+\alpha(\log 4-3-6 \gamma-\xi) \\
& -4 \alpha \log \alpha+2(1-\alpha) \log (1-\alpha)-2 \log (1+\tilde{\Delta}) \\
& +(2-4 \alpha) \log (2+\tilde{\Delta})+2 \log (3+\tilde{\Delta}) \\
& -2(1-\alpha) \log (4+\tilde{\Delta}) \\
& +2 \eta \alpha^{3} \tilde{\Delta}^{2} / \gamma_{z}^{2}-(2-6 \alpha) \log \left(\gamma_{z}\right) .
\end{aligned}
$$

Since $\tilde{\Delta}$ is assumed to be small, we can neglect the $2 \eta \alpha^{3} \tilde{\Delta}^{2} / \gamma_{z}^{2}$ term; in this case we can solve the equation

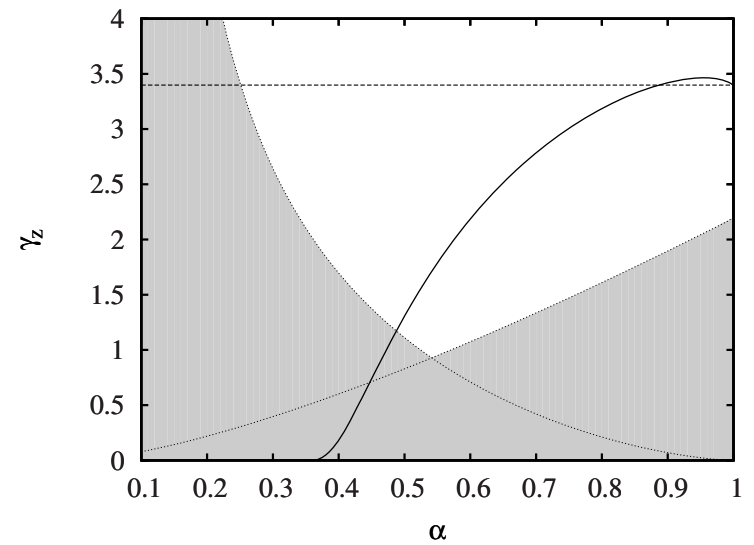

Fig. 3. Theoretical predictions for $\alpha$ and $\gamma_{z}$. The gray area marks the values for $\alpha$ for which either the inner particle line (right of the peak) or the outer one (left of the peak) is unstable. The solid line marks the local extrema $\gamma_{z}^{\text {ext }}(\alpha)$ of $\Delta \tilde{E}\left(\alpha, \gamma_{z}\right)$. For $\gamma_{z}$ above $\approx 3.4$ (dashed line), either two values of $\alpha$ are local extrema, or none.

easily for $\gamma_{z}$, which gives

$$
\begin{aligned}
\gamma_{z}^{\text {ext }}= & \exp [(1+2 \gamma+\alpha(\log 4-3-6 \gamma-\xi)-4 \alpha \log \alpha \\
& +2(1-\alpha) \log (1-\alpha)-2 \log (1+\tilde{\Delta}) \\
& +(2-4 \alpha) \log (2+\tilde{\Delta}) \\
& +2 \log (3+\tilde{\Delta})+(2 \alpha-2) \log (4+\tilde{\Delta})) /(6 \alpha-2)]
\end{aligned}
$$

Figure 3 shows the local extrema $\gamma_{z}^{\text {ext }}$ in the case $\tilde{\Delta}=$ 0 ; qualitatively, the curve looks similar for other values of $\tilde{\Delta}$. For $\gamma_{z}$ below $\approx 3.40$, there is only one $\alpha$ extremal for given $\gamma_{z}$, which is a local a minimum. From $\approx 3.40$ to $\approx 3.45$, a second local extremum appears; the smaller $\alpha$ is a local minimum, the larger $\alpha$ a maximum. Above $\approx 3.45$, there are no local extrema; with decreasing $\alpha$, the curve grows monotonously.

However, the local minimum is not always a global minimum, as can be seen from Figure 4. $\Delta \tilde{E}$ is only smaller than zero for $\alpha<\alpha^{\max } \approx 0.93$, and only these local extrema are also global minima at fixed $\gamma_{z}$. For $\gamma_{z}>\gamma_{z}^{\text {crit }} \approx 3.451$ no such extremum exists; in this case, the energy is minimal for $\alpha=1$.

Below $\gamma_{z}=\gamma_{z}^{\min } \approx 1.175$ the free minimum lies outside of the stability region for the planar configuration, hitting the limit for the stability of the outer particle-rods first. In this case, our theoretical approximations do not apply anymore. However, one can expect that with further decreasing $\gamma_{z}$, also the inner particle-rod breaks up, and the charges form a Wigner-Seitz lattice on the macroion-rod surfaces.

Since $\tilde{\Delta}=0$ in the unconstrained ground state, the only parameter left in the system is $\gamma_{z}$ (see Eq. (1)). Summarizing our results, we obtain the following picture of the system configurations for different values of $\gamma_{z}$ :

- For $\gamma_{z} \geq \gamma_{z}^{\text {crit }}, \alpha=1$ is the ground state, so that the particles are lined up in the center of the gap between the rods. 


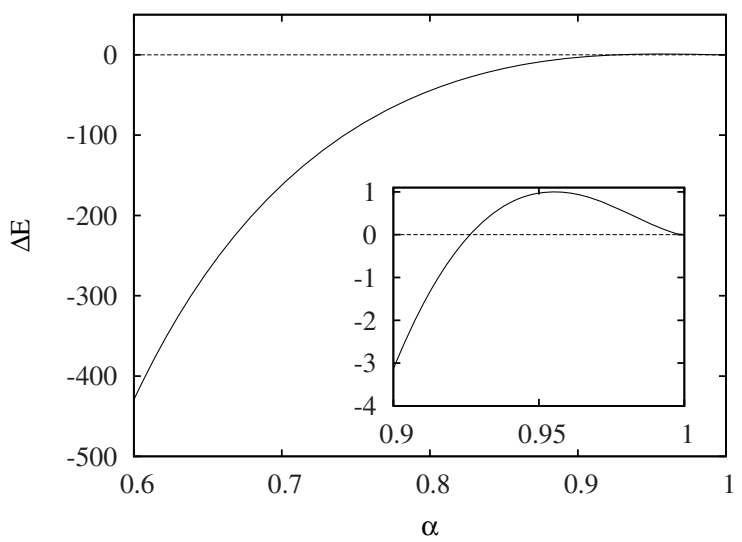

Fig. 4. Energy difference in the local extremum $\Delta E\left(\gamma_{z}^{\text {ext }}\right)$ in arbitrary units. The inset shows that for $\alpha$ larger than $\alpha_{\max } \approx$ 0.93 , the local extremum has a higher energy than $\alpha=1$, for which $\Delta E=0$.

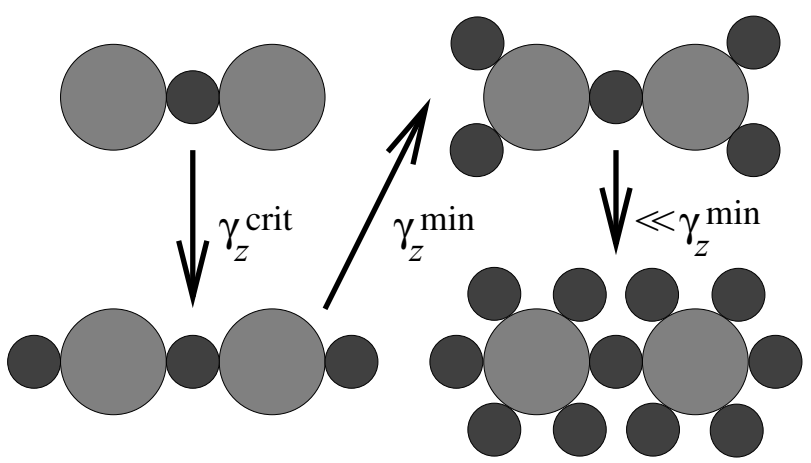

Fig. 5. Cartoon of the different ground-state types developed with decreasing $\gamma_{z}$. Particles are represented by dark gray circles, the macroion-rods by light gray ones.

- For $\gamma_{z}^{\text {crit }}>\gamma_{z}>\gamma_{z}^{\text {min }}$, the optimal $\alpha$ value is smaller than one, however, the particles are still located in the rod-rod plane. The particles arrange in two counterionrods on the outside and one counterion-rod on the inside.

- Below $\gamma_{z}^{\min }$, the outer counterion-rod breaks up first, while the inner particle-rod is still stable. Eventually, with further decreasing $\gamma_{z}$, the charges form a WignerSeitz lattice on the macroion-rod surfaces.

These qualitatively different ground-state types are sketched in Figure 5.

\section{Simulation results for $\mathrm{T}=0$}

As an alternative approach, we have performed computer simulations using the ESPResSo simulation package [30]. Our system consists of 5 -valent counterions and two rods with a line charge density of $\tau=1 / 2$ each; the latter means that all lengths are measured in units of $\lambda=e /(2 \tau)$, where $e$ is the unit charge ${ }^{1}$. The rod radius was varied between 2 and 10 in these units to measure different $\gamma_{z}=q /(\tau R)$ in the range $1 \ldots 5$, and the surface-to-surface

\footnotetext{
1 For DNA, for example, $\lambda=0.17 \mathrm{~nm}$.
}

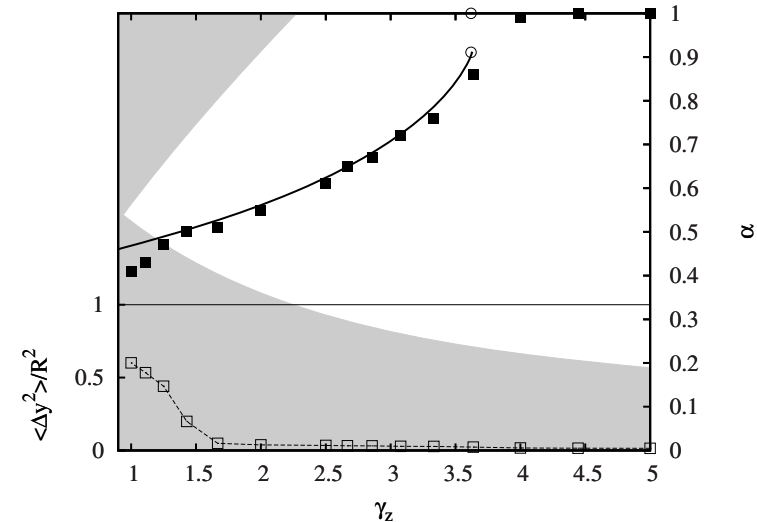

Fig. 6. Simulation results for $\tilde{\Delta}=0.025 \gamma_{z}$. The filled squares denote the $\alpha$ value we find in the approximate ground state, the open squares in the lower half of the graph show the average squared distance $\left\langle\Delta y^{2}\right\rangle$ of the particles to the rod-rod plane in multiples of the rod radius $R$. The gray area again denotes values of $\alpha$ for which either the inner or outer particle-rod gets unstable. The continuous curve marks the prediction for the optimal alpha; the two big open circles mark its discontinuity at $\gamma_{z}^{\text {crit }}$.

distance of the two rods was fixed at $\Delta=1 / 4 \lambda$, which gives $\gamma_{\mathrm{RB}}=40$. To prevent the counterions from escaping at higher temperatures, the system was confined by a cylinder of diameter $4 D$, where $D=2 R+\Delta$ denotes the center-to-center distance of the two macroion-rods.

For the calculation of the electrostatic interactions, the MMM1D method was used. To obtain an infinite system, we introduce periodic boundary conditions along the rods, such that each periodic image contains $N=96$ charges. By repeating some simulations with higher values of $N$, we verified numerically that our findings are not sensitive to the number of charges in each periodic image. A shifted Weeks-Chandler-Anderson potential [31] was used to approximate the hard-core interactions between the rods and the counterions. We used parallel tempering [32] with 48 exponentially distributed temperatures to simulate the system at various temperatures such that the Bjerrum length $l_{\mathrm{B}}$ varied between $0.25 \lambda$ and $1000 \lambda$.

In our simulations, we found $\alpha$ to be practically independent of the temperature for Bjerrum lengths larger than $10 \lambda$. Therefore, it is reasonable to compare the simulation results for $l_{\mathrm{B}}=1000 \lambda$ to our theoretical predictions for the ground state. Figure 6 shows these results for $\alpha$ and the average distance of the particles to the rod-rod plane. Above $\gamma_{z} \approx 1.5$, this distance is practically zero, showing that the particles are indeed located in the plane spanned by the two rods. The values for $\alpha$ are in nice agreement with our theoretical predictions. Moreover, we observe in the simulations the predicted behavior as follows:

- For $\gamma_{z}>3.5 \approx \gamma_{z}^{\text {crit }}$, the configurations are planar with $\alpha=1$, i.e. the counterions line up in the gap, which agrees with the $\mathrm{SC}$ prediction.

- For $\gamma_{z}^{\min } \approx 1.5<\gamma_{z}<3.5$, the configurations are still flat. However, ions are found both between the rods and on the opposite side, see Figure 7. 


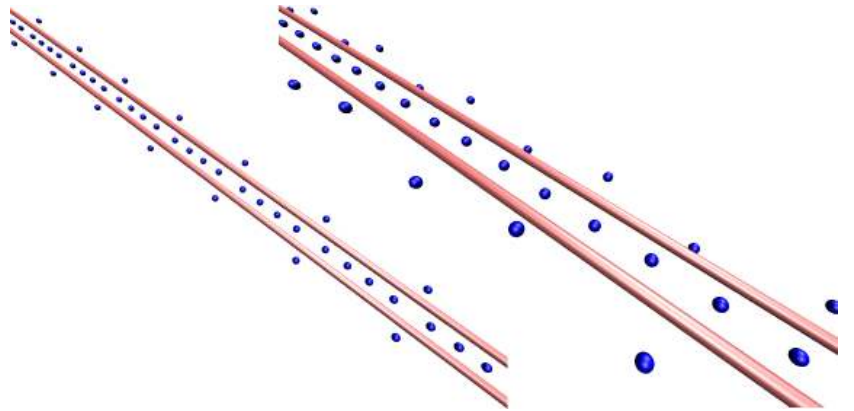

Fig. 7. Snapshots of annealed ground states obtained from the simulations for $\gamma_{z}=2.85$ (left) and $\gamma_{z}=1.43$ (right). Spheres represent the point-like ions; the two rods are displayed much thinner than they really are.

- Below 1.5, the outer counterion-rod breaks up first, while the inner particle-rod is still stable. In the graph one can only see the non-planarity through $\left\langle\Delta y^{2}\right\rangle$, but a visual inspection shows that indeed first the outer particle-rod breaks up into two particle-rods above and below the rod-rod plane, see Figure 7.

\section{Systems with finite temperature}

We will now investigate how the two-rod system behaves at small, but finite temperatures, and up to which temperature our $T=0$ results are still meaningful. In addition, we will compare our results to the strong coupling (SC) theory of Naji, Moreira and Netz [23,24,26]. Also in our $\mathrm{SC}$ calculations, we confined the two rods in a cylinder of diameter $4 \times D$, as we did in our computer simulations. The $\mathrm{SC}$ theory becomes exact in the limit of infinite counterion correlations, i.e., when the strong-coupling parameter $\Xi$ is infinite. For rods the coupling parameter is $\Xi=q^{3} l_{\mathrm{B}}^{2} \tau / R$; therefore the theory is capable, but not limited to systems with a high Bjerrum length or low temperature, and should be well suited to describe our simulation results.

The convergence properties of the SC expansion are however not known in the case of two rods. Previous computer simulations [26] have demonstrated that the $\mathrm{SC}$ results agree with simulations only if the RouzinaBloomfield parameter $\gamma_{\mathrm{RB}}=q /(\tau \Delta)$ is large. The reason for this is that in the SC limit, counterion correlations perpendicular to the rods are negligible compared to those parallel to the rods; therefore the theory is only suited for finite systems in which the parallel correlations dominate. A similar result has been previously found also in the case of two charged plates [33].

Our $T=0$ calculations and the SC theory represent two qualitatively different limits: the $T=0$ limit corresponds to the limit $\Xi \rightarrow \infty$ at constant $\gamma_{z}$, while the strong-coupling limit is $\Xi \rightarrow \infty$ at constant Manning parameter $\xi=q l_{\mathrm{B}} \tau$, which measures the interaction strength of the counterions with the rods. Because $\Xi=\xi^{2} \gamma_{z}$, one necessarily takes the limits $\Xi \rightarrow \infty$ and $\gamma_{z} \rightarrow \infty$ simultaneously (compare Fig. 8). This means that the SC theory is not suited to describe systems with small $\gamma_{z}$. In fact, the

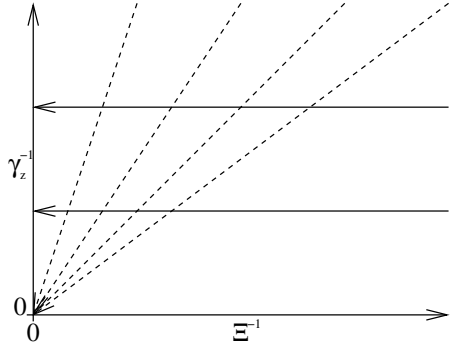

Fig. 8. Schematic view of the $T=0$ and strong-coupling limits. The solid arrows give the direction of the $T=0$ limit, the dotted arrows the direction of the strong-coupling limit. The angle is determined by the Manning parameter $\xi$.

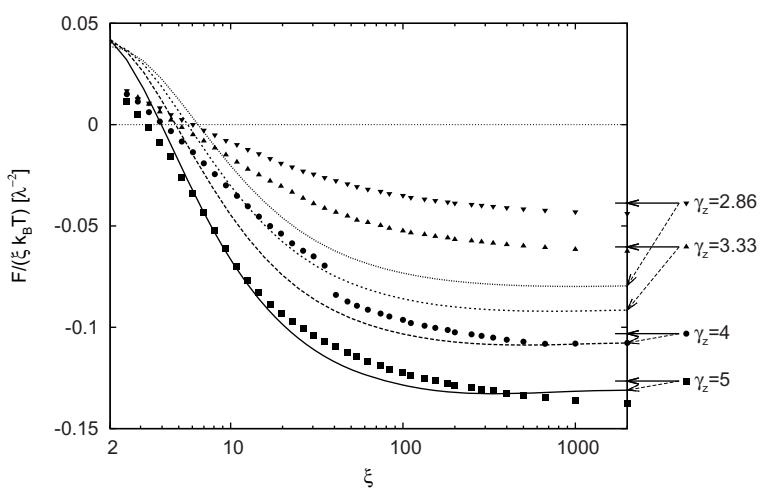

Fig. 9. Simulation results for the effective force per box length between the two rods as a function of the Manning parameter $\xi$. The solid arrows on the right side mark the predictions for the force obtained from equation (10) at the optimal $\alpha$ predicted by equation (16). The dotted arrows point to the corresponding $\mathrm{SC}$ force curves.

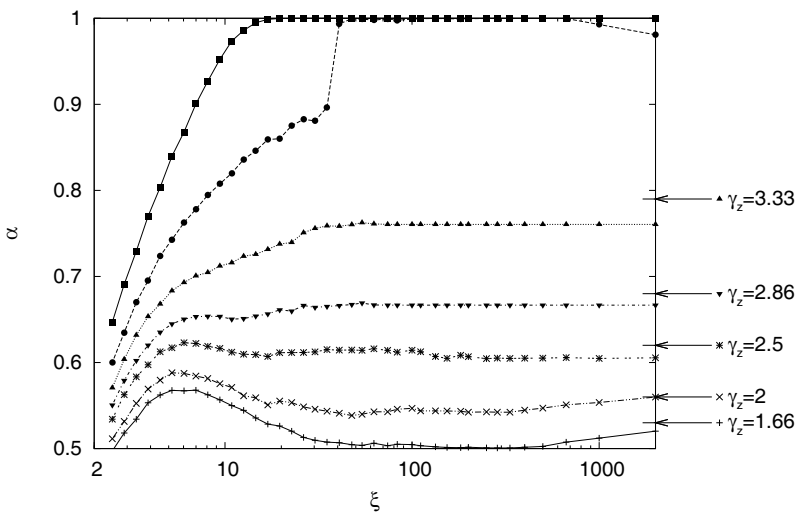

Fig. 10. Simulation results for $\alpha$ as a function of the Manning parameter $\xi$. The solid arrows on the right side mark the predictions for the optimal $\alpha$ obtained from equation (16). For $\gamma_{z} \geq 4$, the prediction is 1 .

SC theory predicts for large Manning parameter a counterion localization in the gap between the rods, which is in agreement with our $T=0$ findings only for large $\gamma_{z}$.

The force between the two rods obtained from our simulations (Fig. 9) agrees indeed reasonably well with the SC force prediction for $\gamma>\gamma_{z}^{\text {crit }}$. This shows that the SC theory is able to correctly predict the tempera- 
ture dependence of the force, which the $T=0$ limit by construction cannot. Only for small Manning parameter and therefore small $\Xi$, the SC curves deviates from the simulation as expected. For $\gamma<\gamma_{z}^{\text {crit }}$, however, the SC force predictions differ significantly from our simulation results, even at large Manning parameters. This can be understood from the particle distribution (see Fig. 10): below $\gamma_{z}^{\text {crit }}$, a significant fraction of the ions is not localized in the gap between the rods, but at the opposite sides of the rods. This effect does not occur in the SC limit, and the theory is therefore not well suited to describe systems with $\gamma_{z}<\gamma_{z}^{\text {crit }}$.

Our $T=0$ predictions, on the other hand, predict the particle distribution correctly even below $\gamma_{z}^{\text {crit }}$, and Manning parameters above 20 (see Fig. 10). Also the force quickly approaches the $T=0$ limit, so that for Manning parameters above 20, the $T=0$ theory is a suitable approximation, although it does not capture the temperature dependence.

\section{Conclusions}

We have presented a method of analytically calculating approximate ground states for a system of two infinite rods and neutralizing counterions. The findings of this analysis were confirmed by computer simulations. In the ground state, the system is characterized by the geometry parameter $\gamma_{z}$, which measures the ratio between the rod radius and the lateral average counterion distance. We have identified several types of ground-state configurations: For $\gamma_{z}>\gamma_{z}^{\text {crit }} \approx 3.45$, the counterions will line up in a single row in the gap between the rods. For $1.18 \approx \gamma_{z}^{\min }<\gamma_{z}<\gamma_{z}^{\text {crit }}$, the particles stay in the plane spanned by the rods, but ions are located both in the gap and on the opposite sides of the rods. For $\gamma_{z}<\gamma_{z}^{\min }$, the internal energy of the particle-rods on the outer surfaces of the rods gets too high, and these particles leave the rod-rod plane.

As an alternative approach, we have performed parallel tempering simulations, which yield not only ground-state approximations, but also configurations at higher temperatures. We compared these configurations both to the strong-coupling (SC) theory of Moreira and Netz [23,24], and to our $T=0$ results. Unlike our zero-temperature calculations, the SC limit provides good predictions for the temperature dependence, however, our $T=0$ predictions cover a wider range of system geometries.

Our simulations suggested parameter limits for both the SC and $T=0$ approaches: the $T=0$ predictions are suitable to describe systems with Manning parameter above 20. Moreover, the $T=0$ approximations require $\gamma_{\mathrm{RB}}>5$, i.e. the rods need to be close, and $\gamma_{z}>\gamma_{z}^{\min } \approx$ 1.18 .

Together with previous simulation studies [26], we find similar, but stronger conditions for the application of the SC theory to the case of two rods: it is a good description for large coupling parameter $\Xi>50, \gamma_{\mathrm{RB}}>5$, and $\gamma_{z}>$ $\gamma_{z}^{\text {crit }} \approx 3.45$. The two last conditions are similar in spirit: they describe the requirement that the average counterion distance parallel to the rods is much larger than the gap size $\left(\gamma_{\mathrm{RB}}\right)$ and the rod size $\left(\gamma_{z}\right)$. In this case, counterion correlations perpendicular to the rods are negligible, which are not captured by the SC approach.

However, we want to emphasize that from an experimental point of view, $\gamma_{z}$ plays a much more important role. While in systems where the rods attract, $\gamma_{\mathrm{RB}}$ is automatically large because the rods approach closely, the value of $\gamma_{z}$ is a constant and determined by the investigated system. For DNA and trivalent counterions (e.g. spermidine), the value for $\gamma_{z}$ is very low, only $\approx 0.5$. Therefore one cannot expect that either our $T=0$ theory nor the SC theory will provide a good quantitative agreement for this system. In fact, for most polyelectrolyte systems and relevant ranges of coupling parameters, $\gamma_{z}$ will be smaller than 3, so that the SC limit appears to be not a good approximation, and our ground-state approximation will also work for large couplings only if $\gamma_{z}>1.18$.

We thank M. Deserno for general comments, A. Naji and R. Netz for many useful discussions regarding the strong-coupling theory, and an anonymous referee on a previous version for helpful remarks. This work has been supported by German Science Foundation (DFG) through SFB 625, TR6 and Ho$1108 / 11-2$, and by the research program of the Stichting voor Fundamenteel Onderzoek der Materie (FOM), which is supported by the Nederlandse Organisatie voor Wetenschappelijk Onderzoek (NWO). AA acknowledges support from the MarieCurie program of the European Commission under the 6th framework program.

\section{References}

1. J.C. Neu, Phys. Rev. Lett. 82, 1072 (1999).

2. E. Trizac, J.L. Raimbault, Phys. Rev. E 60, 6530 (1999).

3. E. Trizac, Phys. Rev. E 62, R1465 (2000).

4. J.E. Sader, D.Y. Chan, J. Colloid Interface Sci. 213, 268 (1999).

5. L. Guldbrand, L.G. Nilsson, L. Nordenskiöld, J. Chem. Phys. 85, 6686 (1986).

6. L.G. Nilsson, L. Guldbrand, L. Nordenskiöld, Mol. Phys. 72, 177 (1991).

7. A.P. Lyubartsev, L. Nordenskiöld, J. Phys. Chem. 101, 4335 (1997).

8. A.P. Lyubartsev, J.X. Tang, P.A. Janmey, L. Nordenskiöld, Phys. Rev. Lett. 81, 5465 (1998).

9. N. Grønbech-Jensen, R.J. Mashl, R.F. Bruinsma, W.M. Gelbart, Phys. Rev. Lett. 78, 2477 (1997).

10. M. Deserno, C. Holm, S. May, Macromolecules 33, 199 (2000).

11. M.L. Bret, B.H. Zimm, Biopolymers 23, 271 (1984).

12. F.J. Solis, M.O. de la Cruz, Phys. Rev. E 60, 4496 (1999).

13. E. Allahyarov, H. Löwen, Phys. Rev. E 62, 5542 (2000).

14. A. Diehl, H.A. Carmona, Y. Levin, Phys. Rev. E 64, 01180 (2001).

15. Y. Levin, Rep. Prog. Phys. 65, 1577 (2002) http:// stacks .iop.org/RoPP/65/1577.

16. C. Guaqueta, E. Luijten, Phys. Rev. Lett. 99, 138302 (2007).

17. J. Widom, R.L. Baldwin, J. Mol. Biol. 144, 431 (1980). 
18. R. Podgornik, D. Rau, A. Parsegian, Biophys. J. 66, 962 (1994).

19. V.A. Bloomfield, Curr. Opin. Struct. Biol. 6, 334 (1996).

20. O. Lambert, L. Letellier, W.M. Gelbart, J.L. Rigaud, Proc. Natl. Acad. Sci. U.S.A. 97, 7248 (2000).

21. W.M. Gelbart, R.F. Bruinsma, P.A. Pincus, V.A. Parsegian, Phys. Today 53, 38 (2000).

22. M. Deserno, A. Arnold, C. Holm, Macromolecules 36, 249 (2003).

23. A.G. Moreira, R.R. Netz, Phys. Rev. Lett. 87, 078301 (2001).

24. A.G. Moreira, R.R. Netz, in Electrostatic Effects in Soft Matter and Biophysics, edited by C. Holm, P. Kékicheff, R. Podgornik, Vol. 46 of NATO Science Series II - Mathematics, Physics and Chemistry (Kluwer Academic Publishers, Dordrecht, Nl, 2001).
25. A. Naji, R. Netz, Eur. Phys. J. E 13, 43 (2004).

26. A. Naji, A. Arnold, C. Holm, R.R. Netz, Europhys. Lett. 67, 130 (2004).

27. W. Earnshaw, Trans. Camb. Philos. Soc. 7, 97 (1842).

28. A. Arnold, C. Holm, J. Chem. Phys. 123, 144103 (2005).

29. M. Abramowitz, I. Stegun, Handbook of Mathematical Functions (Dover Publications Inc., New York, 1970).

30. H.J. Limbach, A. Arnold, B.A. Mann, C. Holm, Comput. Phys. Commun. 174, 704 (2006).

31. J.D. Weeks, D. Chandler, H.C. Andersen, J. Chem. Phys. 54, 5237 (1971).

32. D. Frenkel, B. Smit, Understanding Molecular Simulation, 2nd edition (Academic Press, San Diego, 2002).

33. H. Boroudjerdi, Y.W. Kim, A. Naji, R.R. Netz, X. Schlagberger, A. Serr, Phys. Rep. 416, 129 (2005). 